\title{
THE OBSERVATIONAL EVIDENCE FOR ACCRETION
}

\author{
LEE HARTMANN \\ Center for Astrophysics, 60 Garden St., \\ Cambridge, MA 01238 USA
}

\begin{abstract}
Outflows from low-mass young stellar objects are thought to draw upon the energy released by accretion onto $\mathrm{T}$ Tauri stars. I briefly summarize the evidence for this accretion and outline present estimates of mass accretion rates. Young stars show a very large range of accretion rates, and this has important implications for both mass ejection and for the structure of stellar magnetospheres which may truncate T Tauri disks.
\end{abstract}

\section{Introduction}

Star formation requires the contraction of typical molecular cloud cores by factors of a million or more to reach stellar densities. The contraction process must involve both redistribution of angular momentum and release of gravitational potential energy to produce a star. The necessary angular momentum transfer seems to occur mainly in circumstellar disks, which allow accretion to a central stellar core as a smaller amount of matter is pushed out (or viscously spread) to larger radii. Much of the energy release appears to take the form of radiation; however, a substantial fraction of the energy generated by accreting material apparently is put into outflows or jets which produce Herbig-Haro objects (see Calvet, this volume). The rapidly-rotating disk probably also plays an essential role in jet ejection by providing ideal conditions for the centrifugal acceleration of material by magnetic fields; the rotating magnetic field structure is most likely responsible for the high collimation observed in many jets (see the contributions by Pudritz \& Ouyed, Shu \& Shang, Camenzind, and Heyvaerts \& Norman in this volume).

A knowledge of mass accretion processes in young stellar objects is essential to developing a fundamental understanding of outflows. Some jets and $\mathrm{HH}$ objects show unmistakable evidence for highly time-dependent out- 


\section{FU Ori object}

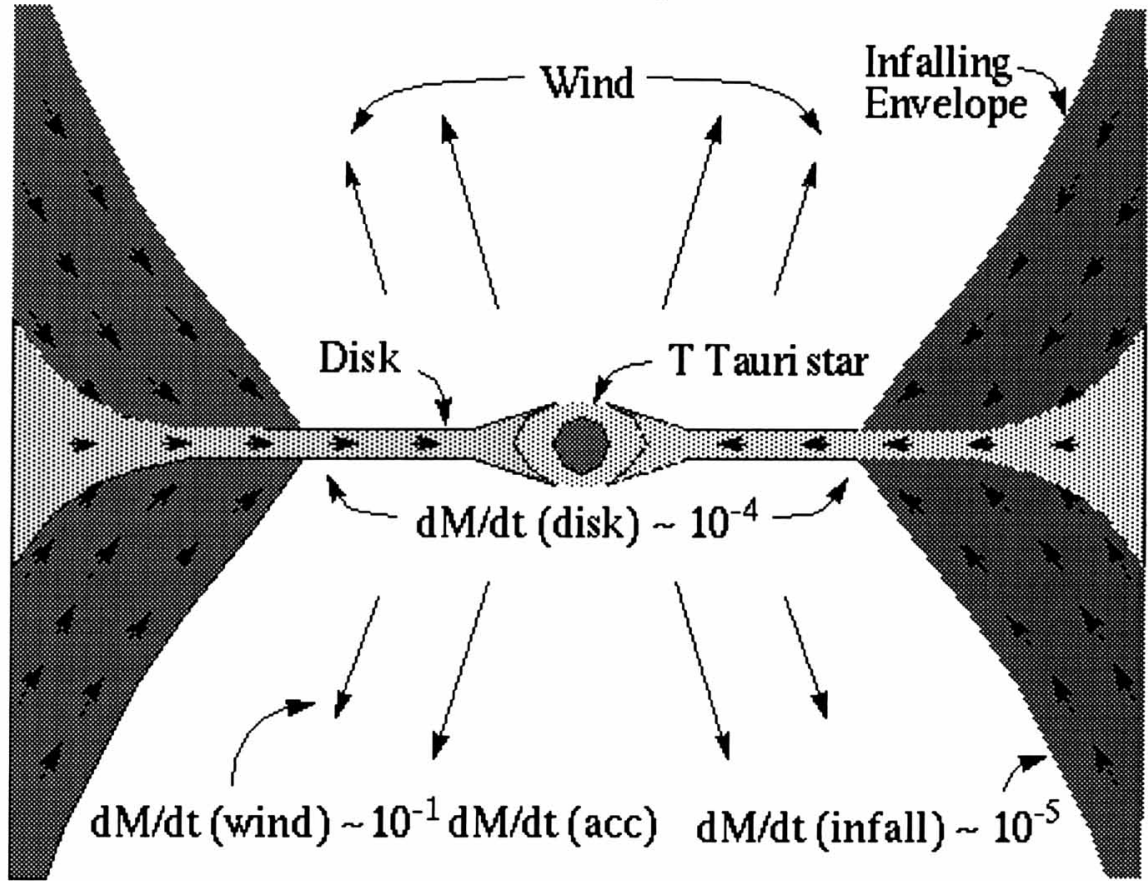

\section{T Tauri star}

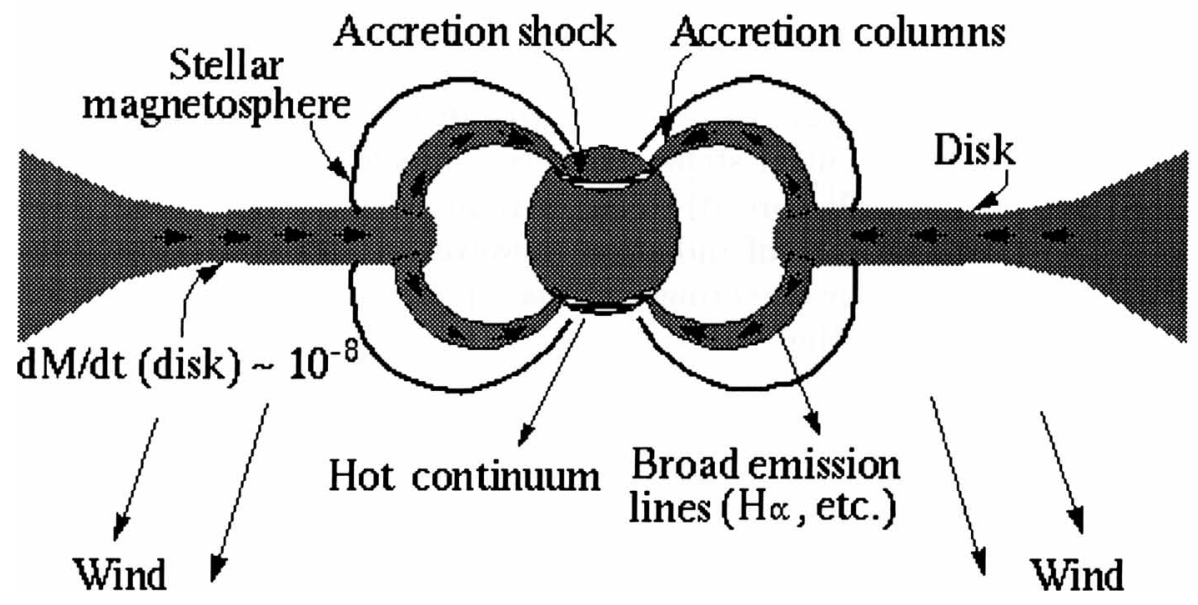

Figure 1. Two main types of accreting pre-main sequence objects (see text). Mass accretion/loss rates are given in units of solar masses per year 
flow (see Bally \& Devine, this volume), and it has been recognized for some time that the time-dependence of mass accretion might be responsible for this structure (Reipurth 1989,1991 ). In addition to the time-variability of accretion, the very large range in accretion rates for objects with outflows provides some important clues to the processes responsible for mass loss.

In this review I shall concentrate on mass accretion in low-mass, premain sequence stars. The subject can be conveniently divided into discussions of two types of objects, the FU Ori eruptive variables and the (more numerous) T Tauri stars. FU Ori objects are very young, probably low-mass stars accreting from circumstellar disks at rates as high as $10^{-4} M_{\odot} \mathrm{yr}^{-1}$ (Figure 1). This rapid accretion is highly time-variable and probably especially prevalent in the earliest stages of star formation, when material from the infalling natal envelope may still be falling onto the outer disk. The $\mathrm{T}$ Tauri stars exhibit much lower mass accretion rates through their disks. Most T Tauri stars are older than FU Ori objects, although it appears quite likely that FU Ori objects oscillate between rapid FU Ori disk accretion and slower, $\mathrm{T}$ Tauri-like accretion.

An important distinction between FU Ori and T Tauri accretion is the effect of the stellar magnetosphere (see Edwards, this volume). It now seems likely that the inner disks of $\mathrm{T}$ Tauri stars are truncated by the magnetosphere, and that accretion to the central star occurs along magnetic field lines, terminating in a hot accretion shock at the stellar surface (Figure 1). In contrast, the FU Ori objects show no evidence for magnetospheric disk truncation, probably because the magnetosphere of the central $\mathrm{T}$ Tauri star is crushed by the high pressures in rapid accretion. Although these objects apparently have very different inner disk boundary conditions, outflows with qualitative similarities are produced in both FU Ori objects and $\mathrm{T}$ Tauri stars, and this fact has implications for understanding mass loss mechanisms.

\section{FU Ori accretion}

The evidence for disk accretion in FU Ori objects has been summarized in a recent review (Hartmann \& Kenyon 1996) and so I will not repeat that material here. In outline, the validity of the accretion disk model for FU Ori objects rests on several observational results: that the spectral energy distributions (SEDs) of FU Ori objects can be modeled reasonably well with steady disk spectra, at least shortward of $10 \mu \mathrm{m}$; that the optical and infrared lines indicate rapid rotation, with doubled line profiles often observed that are expected for a rotating disk; and that the differential rotation observed as a function of wavelength (slower rotation at longer wavelengths) is consistent with quasi-Keplerian disk rotation. The obser- 
vation of absorption lines in FU Ori objects is consistent with the rapid accretion model; at high accretion rates and optical depths, the viscous energy dissipation is trapped in the disk midplane, helping to create the outwardly-decreasing temperature gradient needed to produce absorption features.

Perhaps the single most important point to be made in the present context is that, during an FU Ori accretion event, the accretion luminosity of the disk far exceeds that of the (original) central star; typical luminosities of FU Ori objects are $200-500 L_{\odot}$, whereas typical T Tauri star luminosities are $\sim 1 L_{\odot}$. This means that the emergent spectrum is dominated by the disk, and can be compared directly with disk models without making direct allowance for radiation of the central star.

The spectral energy distributions (SEDs) of FU Ori objects can be modeled by pure disk models, without including any hot radiating components such as are present in T Tauri stars (Kenyon et al. 1989). The implication is that FU Ori objects have neither the canonical boundary layer between rapidly rotating star and disk (e.g., Lynden-Bell \& Pringle 1974) nor the hot magnetospheric accretion shock thought to be present in $\mathrm{T}$ Tauri stars (Königl 1991; §3). Both constraints are explicable in terms of the rapid disk accretion rates in FU Ori objects.

The absence of a magnetosphere can be explained simply by the pressure of rapid accretion, as discussed in more detail in $\S 4$. The absence of boundary layer emission can also be understood by the following argument. For plausible viscosities, the disks of FU Ori objects are likely to be extremely optically thick (Clarke et al. 1989; Clarke et al. 1990; Bell et al. 1995), resulting in very strong trapping of the internal viscous dissipation of accretion energy. This in turn leads to very high central disk temperatures in the innermost regions, such that the internal sound speed is an appreciable fraction of the local Keplerian velocity (Clarke et al. 1989; Popham et al. 1993). Under these conditions, boundary layers are unlikely to form for some combination of the following reasons. First, since the disk is physically thick, with a ratio of scale height $H$ to cylindrical radius $R$ of $H / R \sim 0.3$, any spatially-concentrated dissipation of energy will be diffused laterally over a region $\Delta R \sim H$, which in turn reduces the maximum temperature observed (Clarke et al. 1989). Second, because of the large optical depths involved, the disk may advect substantial amounts of energy (Clarke et al. 1989); this energy may actually be carried into the central star rather than radiated, possibly explaining why the inner radii of FU Ori disks seem to be a factor of two larger than typical T Tauri star radii (Popham et al. 1993, 1996). Third, gas pressure support in the radial direction is no longer negligible; this means that the disk rotation can be substantially sub-Keplerian over a finite radial distance, implying dissipation of rotational energy over 
a much larger region of the disk than in a typical boundary layer, and this spreading of dissipating energy also helps prevent the high temperature emission characteristic of boundary layers (Popham et al. 1996).

Accretion rates for FU Ori objects are subject to several uncertainties, in part because of the relatively unknown properties of the central stars. The disk accretion luminosity

$$
L_{a c c}=\frac{G M_{*} \dot{M}_{a c c}}{(2) R_{*}}
$$

can be used to derive the mass accretion rate $\dot{M}_{a c c}$ if the stellar mass $M_{*}$ and radius $R_{*}$ are known. The radius can be estimated from the luminosity and peak temperature of disk emission, the latter determined from spectral type matching. In principle, once the radius is known, the mass can be estimated from the rotational velocity, given an inclination; however, application of this method to the FU Ori objects V1057 Cyg and V1515 Cyg indicate very small central masses unless these disks are observed nearly pole-on - which they probably are (Goodrich 1987). In general the inclination uncertainties are large, and it is probably better to simply assume a typical T Tauri stellar mass. The factor of 2 in the denominator of equation (1) is the standard accretion disk result, which assumes that any additional energy release occurs in a boundary layer. As discussed above, there is no boundary layer emission in FU Ori objects, but the factor of 2 remains uncertain because it is not known how much of the accretion energy of the innermost disk is directly radiated and how much is advected into the star.

Within these uncertainties, observations indicate that FU Ori objects must have accretion rates of order $\dot{M}_{a c c} \sim 10^{-4} M_{\odot} \mathrm{yr}^{-1}$ at maximum light. This extremely rapid accretion is accompanied by very strong mass ejection from the surface of the disk (Calvet, this volume).

The outburst behavior of FU Ori objects is especially interesting in view of the evidence for multiple jet outbursts (Reipurth 1991; Bally \& Devine, this volume). This is very difficult to come by, because we have never observed long enough to see multiple outbursts, or a complete cycle, or even a return to minimum light in any FU Ori object. Event statistics (Hartmann \& Kenyon 1996) suggest that the typical low-mass star may undergo $5-10$ FU Ori outbursts in its lifetime, assuming that we have detected all FU Ori events within $1 \mathrm{Kpc}$ of the Sun over the last 50 years. If we then assume that FU Ori outbursts are confined to the protostellar infall phase, which probably lasts $\sim 10^{5} \mathrm{yr}$, we find a timescale between outbursts of $\sim 10^{4}$ yr. If instead the detection statistics are seriously incomplete, so that most of the mass of a typical low-mass star is accreted in FU Ori events, then the frequency increases by a factor of 5 , the repetition timescale of $\sim 2000$ yr appears to be more in line with observed multiple bow shock structures 
in some jets. My personal guess is that there is a large range in outburst behavior among pre-main sequence stars and FU Ori objects represent one extreme; and that milder, more frequent events, perhaps analogous to the "EXor" outbursts of Herbig (1977), are responsible for the multiple jet bow shocks in most cases.

\section{T Tauri accretion}

The T Tauri stars have much lower disk accretion rates than FU Ori objects, typically by factors $\sim 10^{3}-10^{4}$ (see below). The low accretion rates mean that it is difficult to distinguish the local accretion energy heating in the disk from energy input of absorbed light from the central star (Figure 1). It is not generally feasible to determine accretion rates from disk emission. Instead, one must rely on measurements of the hot continuum emission from the accretion shock region (Figure 1).

Several authors have presented estimates of accretion rates using the hot continuum emission of T Tauri stars (Bertout, Basri, \& Bouvier 1988; Basri \& Bertout 1989; Hartigan et al. 1990, 1991; Valenti, Basri, \& Johns 1993; Hartigan, Edwards, \& Ghandour 1995). These authors analyzed the hot continuum emission in terms of a boundary layer model, in which the accretion luminosity is related to the mass accretion rate essentially as in equation (1). In the current interpretation of $\mathrm{T}$ Tauri emission, material from the disk moves to the star in essentially free-fall from its starting point at the magnetospheric radius $R_{m}$. Since the rotational velocities of $\mathrm{T}$ Tauri stars are small, one expects the hot continuum emission represents the dissipation of the kinetic energy generated by material falling in from $R_{m}$; hence,

$$
L_{h o t} \sim \frac{G M_{*} \dot{M}_{a c c}}{R_{*}}\left(1-\frac{R_{*}}{R_{m}}\right) .
$$

While this method appears straightforward in principle, in practice there are problems in evaluating $L_{h o t}$ which basically result from the need to separate out the stellar photospheric emission from the accretion shock emission, and to make an appropriate extinction correction. Because the hot continuum is bluer than a typical $\mathrm{T}$ Tauri star photosphere, extinction corrections which do not take the added continuum into account may underestimate the extinction, and since much of the excess emission appears in the Balmer continuum, reddening corrections are important. In addition, the Paschen continuum emission is also an important contributor to the total accretion luminosity in many systems, and evaluating this requires careful separation between stellar and accretion shock radiation. As an indication of how difficult the problem is, two recent careful studies, concentrating on differing spectral regions, and making differing assumptions 
for analysis, differ systematically by an order of magnitude in the inferred hot continuum luminosities and mass accretion rates (Valenti, Basri, \& Johns 1993; Hartigan, Edwards, \& Ghandour 1995).

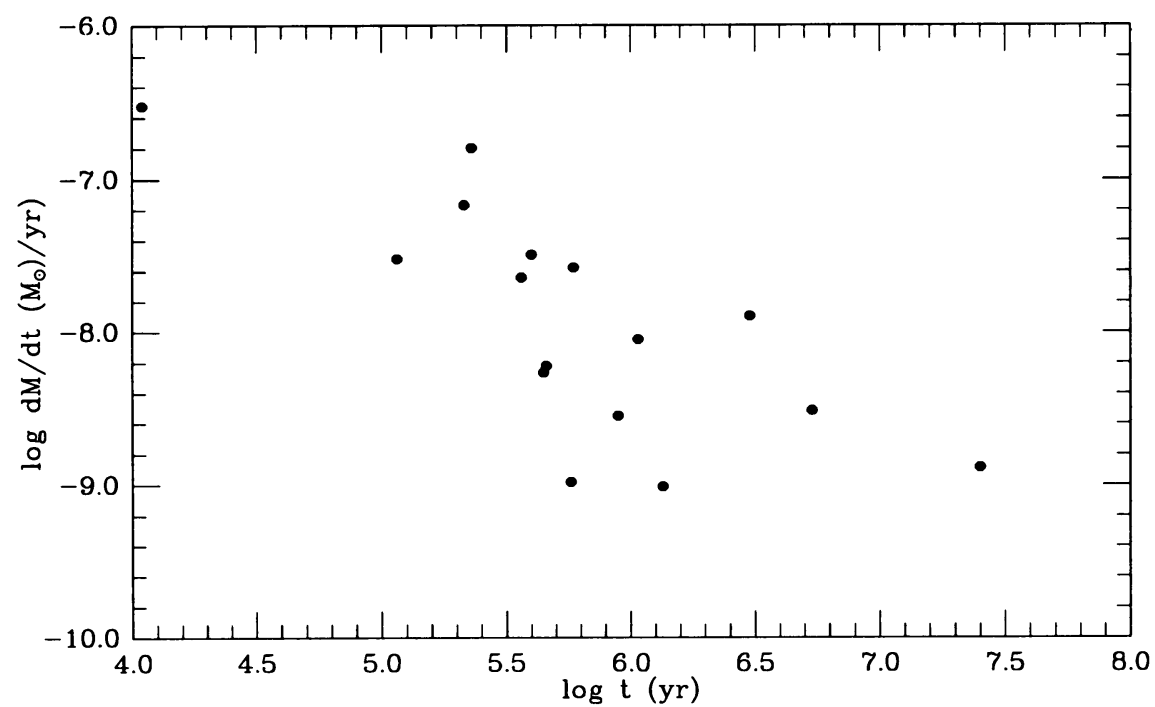

Figure 2. Mass accretion rates for representative $\mathrm{T}$ Tauri stars in the Taurus molecular cloud (see text). From Gullbring et al. (1997)

Because of this large systematic discrepancy, and because of the importance of determining mass accretion rates for $\mathrm{T}$ Tauri stars, we decided to reexamine this question. Our method (Gullbring et al. 1997) employs elements of both previous works. We obtained intermediate resolution absolute spectrophotometry of a small sample of Taurus classical T Tauri stars spanning the wavelength range $3200 \AA$ to $5200 \AA$. Thus, we concentrate on the Balmer and upper Paschen continuum emission, like Valenti et al., but we tried to measure the absorption features at intermediate resolution, in the spirit of the high-resolution method of Hartigan et al., to separate between star and hot "veiling" continuum.

Our results, shown in Figure 2, are generally closer to the results of Valenti et al. with substantial scatter. We think that we understand the sources of the discrepancy; basically, Hartigan et al. assumed that the Paschen continuum is stronger than it generally is, and this causes an overestimate of the amount of emission at wavelengths outside of the region of observation, as well as producing a bias toward overestimating the extinc- 
tion. In addition, there is nearly a factor of two systematic difference in determining mass accretion rates from luminosities using equation (2) (assuming $R_{m} \sim 5 R_{*}$ ) rather than using the usual boundary layer equation, as Hartigan et al. assumed.

The results in Figure 2 indicate a wide range in accretion rates among $\mathrm{T}$ Tauri stars. The data are biased against high accretion rate stars, because the extinction corrections are difficult to determine for these objects, and so both the accretion luminosities and stellar ages are difficult or impossible to determine; thus, the true spread in accretion rates is larger than indicated. There is some evidence for a general decline in mass accretion rates as a function of age, as might be expected if accretion rates depend in part upon the mass remaining in the disk.

\section{Implications for magnetospheres and wind ejection}

The foregoing discussion indicates that instantaneous mass accretion rates in low-mass stars span a wide range, from up to $\sim 10^{-4} M_{\odot} \mathrm{yr}^{-1}$ in FU Ori events to as low as $\sim 10^{-9} M_{\odot} \mathrm{yr}^{-1}$ in slowly-accreting $\mathrm{T}$ Tauri stars. There is evidence for both short-term time variability as well as a general decline in accretion rates as a function of age. In addition to the dramatic variations during FU Ori outbursts, significant changes in accretion onto the central $\mathrm{T}$ Tauri star have been observed in classical T Tauri stars. For example, the (currently) strong-emission star DR Tau increased in optical brightness (B magnitude) by about a factor of ten over a period of approximately 10 years (Herbig 1989). Less is known about the variability of accretion in most $\mathrm{T}$ Tauri stars. The observed range in mass accretion rate as a function of age in Figure 2 suggests that this variability might be as much as an order of magnitude, but this is likely to be an upper limit given uncertainties in stellar ages and in measuring accretion luminosities, resulting from errors in extinction estimates, differing geometric projections of the accretion shock along the line of sight, rotational modulation of nonaxisymmetric magnetospheric structure, etc.

Figure 3 summarizes the current observational constraints. This figure should be taken as illustrative rather than conclusive. The ages and timescales of the accretion phases are not well known; furthermore, they may overlap. For example, the early phases of accretion probably oscillate between FU Ori outbursts and more typical T Tauri accretion. However, viewed broadly, Figure 3 usefully indicates the wide range of accretion rates present in low-mass stars, and that the high accretion rate phases are probably concentrated to earlier times.

What are the implications of this behavior for wind/jet ejection? In general, we expect that accretion provides the energy for mass ejection 


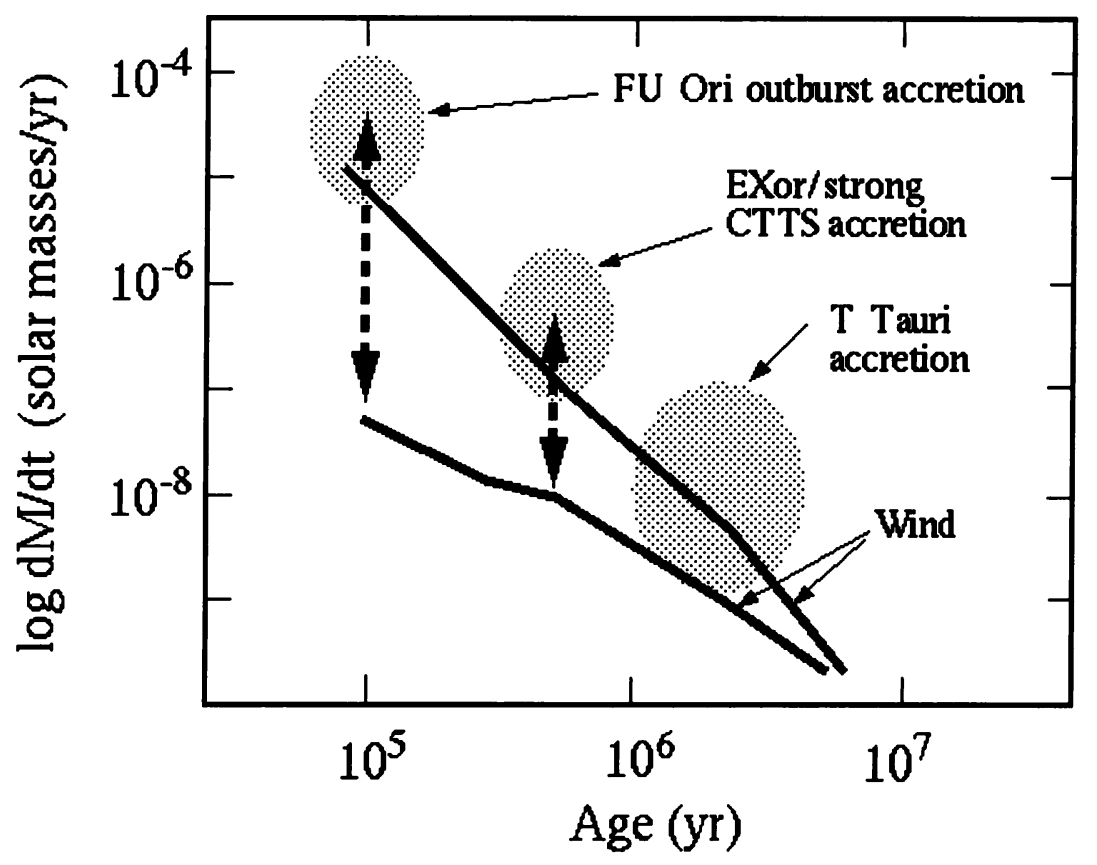

Figure 3. Highly schematic picture of accretion in low-mass stars. FU Ori outbursts are thought to occur mostly at early phases of evolution; the data in Figure 2 suggest that disk accretion rates generally decrease with increasing age. Wind mass loss rates generally should fall below accretion rates for energetic reasons (see text), although the time-variability of accretion, as typified at its extreme end by FU Ori objects, complicates the issue.

(e.g., Shu \& Shang, this volume). Therefore, unless there is $100 \%$ efficiency of conversion of accretion energy to mass loss energy (which seems unlikely; Hartmann 1995), the kinetic energy flux in the wind must be a fraction of the total accretion energy. It is more useful to consider the mass flux in the wind. In a (quasi) steady state, the energy supplied to the wind or jet comes from the potential energy released as mass moves from infinity down to some radius in the potential well. The mass ejected must escape from this gravitational potential well, and have some extra energy besides to have a finite escape velocity at infinity. Thus, even in the limit where all of the accretion energy goes into accelerating the wind, there must be less mass ejected (at higher energy) than is accreted (at lower energy). Since wind velocities are typically a few times the escape velocity at the point from which mass is ejected, and since not all of the accretion energy goes 
into the wind (some is radiated away), one might expect that the maximum mass loss rates in winds/jets driven by disk accretion are at most of order $10^{-1}$ of the mass accretion rates. This is rougly what is observed (Calvet, this volume), which indicates pretty high efficiencies of converting accretion energy into wind energy.

If we take mass loss rates to be $10^{-1}$ of accretion rates, then we have the curves marked "wind" in Figure 3. The curves must span a range of mass loss rates to accomodate the accretion variability at a given time. Again, one must take these wind limits as schematic rather than highly accurate. Evolutionary timescales are likely to vary from star to star. Given these caveats, Figure 3 makes an important point; namely, that wind ejection is likely to decline steeply with age, and that in terms of impact on the interstellar medium, outbursting/rapidly-accreting young stellar objects are likely to dominate. We should be wary of trying to interpret outflow dynamics in terms of the mass ejection behavior of the most common $\mathrm{T}$ Tauri stars, because these objects probably do not dominate the time-integrated outflow properties.

Another way of looking at this is to consider the amount of mass accreted in various phases. One may estimate the mass accreted during the "typical" " T Tauri phase (which I define here as representing ages $\gtrsim 10^{6}$ yr) from the results in Figure 2;

$$
M_{a c c} \sim \dot{M}(t) \times t \sim 10^{-8} M_{\odot} \mathrm{yr}^{-1} \times 10^{6} \mathrm{yr} \sim 10^{-2} M_{\odot} .
$$

This means that most of the mass of a typical T Tauri star of mass $\sim 0.5 M_{\odot}$ must be accreted at earlier ages. If we assume a more or less universal ratio of $\dot{M}_{\text {wind }} \sim 10^{-1} \dot{M}_{a c c}$, this suggests that over $90 \%$ of the outflow mass ejected occurs at ages $<10^{6} \mathrm{yr}$. Moreover, the ram pressure of the flow is also an important quantity in understanding the driving outward of sweptup interstellar medium; this places even more emphasis on possible shortlived events of very rapid mass loss, such as are likely to be associated with outbursts of accretion.

These considerations emphasize the point that "typical" accreting $\mathrm{T}$ Tauri stars should not necessarily be taken as the model for outflow sources. It is fairly certain now that stellar magnetospheres play a crucial role in inner disk structure in most T Tauri stars (Königl 1991; Camenzind 1990; see Edwards, this volume). However, FU Ori objects do not show the observational signatures with magnetospheres, and indeed at the high accretion rates during FU Ori objects, one might well expect the ram pressure of the disk to overwhelm the stellar magnetosphere (Shu et al. 1994). Thus it seems to me that the emphasis on the importance of the stellar magnetosphere producing mass ejection in the picture of Shu and collaborators (Shu et al. 1994) may be misplaced. 
An issue which is indirectly related to mass accretion and ejection is the location of the magnetospheric radius in $\mathrm{T}$ Tauri stars with respect to the co-rotation radius. Magnetic fields which connect to disk regions outside of the co-rotation radius, i.e. to parts of the disk which have a slower angular velocity than that of the star, will help spin down the star by transferring angular momentum outward. However, this angular momentum transfer implies that accretion is not occurring along these field lines. In contrast, stellar magnetic field lines connecting to the disk interior to the corotation radius attach to parts of the disk with higher angular velocities than the star; in this case, the magnetic field lines transfer angular momentum from the Keplerian disk to the star, allowing infall to proceed.

The problem is that we wish to have both accretion and stellar spindown. In view of the observation that all $\mathrm{T}$ Tauri stars with near-infrared disk emission exhibit accretion diagnostics (Edwards et al. 1994), it appears that nearly all disks of any significance are accreting; on the other hand, it appears that the accreting $\mathrm{T}$ Tauri stars are more slowly rotating than the non-accreting T Tauri stars without disks (Bouvier et al. 1993; Edwards et al. 1993). This suggests that disks serve as a rotational brake (e.g., Königl 1991), which happens if magnetic field lines connect to the disk exterior to corotation, at the same time that accretion proceeds, which requires the stellar magnetosphere to connect with disk regions interior to corotation.

The original magnetosphere model of Ghosh \& Lamb (1979) solved this problem by having the disk penetrated by a range of magnetic field lines, both interior and exterior to the corotation radius. However, there are difficulties with this model. It is not apparent that the dynamics of the disk can be significantly affected over a wide range of disk radii (Wang 1995, 1996); and there is no guarantee that the magnetic field lines can easily slip through the disk at just the right rate to produce a steady-state configuration (Shu et al. 1994).

For these reasons Shu et al. (1994) developed a model in which the magnetosphere is truncated at the disk at exactly the corotation radius. This model is attractive in that it avoids both problems of the Ghosh and Lamb model, and provides a definite inner boundary condition which can be used to calculate the details of the accretion flow and the mass ejection (Ostriker \& Shu 1995; Najita \& Shu 1994; see Shu \& Shang, this volume). However, this solution is obtained at what may be an unacceptable price, namely that the magnetospheric radius is exactly at corotation. For the case of a dipole field, we expect the magnetospheric radius to scale as

$$
R_{m} \propto \dot{M}_{a c c}^{-2 / 7} B_{*}^{4 / 7}
$$

(cf. Königl 1991), where $B_{*}$ is the stellar (dipole) magnetic field. Given the wide range of mass accretion rates in low-mass young stellar objects, and 
the evidence for some (rapid) time variability, it is not at all clear that the stellar magnetic field will always truncate the disk at the corotation radius. For example, in DR Tau, the accretion rate seems to have increased by an order of magnitude over a period of a decade, which is far too short for the stellar rotation rate to change. Thus, unless the stellar magnetic field changed in precise proportion to the change in disk accretion rate which seems implausible - an order of magnitude change in $\dot{M}_{\text {acc }}$ would yield a change in $R_{m}$ of a factor of nearly 2, corresponding to a change in the Keplerian angular velocity of the intercepted disk regions of a factor of roughly 2.7. The significance of this result can be emphasized by noting that even if the angular velocities of the star and the disk region where a magnetic field line is attached match to $10 \%$. the field line would still wind up over about 10 rotations - only about 10 weeks for the typical $\mathrm{T}$ Tauri star.

It seems necessary to relax the criterion that the magnetospheric truncation occurs precisely at corotation. Indeed, as pointed out by Shu \& Shang (this volume), the evidence for non-axisymmetric magnetic field structure implies that not all portions of the truncated disk can be at the corotation radius. It may well be that, under differing conditions of accretion, the central $\mathrm{T}$ Tauri stars have a net spin up or spin down torque at any given instant of time, and that an equilibrium is achieved only over relatively long timescales.

Even with this assumption, we still need to consider the likely winding up of field lines in the stellar magnetosphere, as emphasized by Shu in this volume. What this might look like was indicated schematically a few years ago by Van Ballegooijen (1994). He considered a very simple case in which the field lines were force-free outside the star and disk, and were simply rooted in disk regions which rotate at a different angular velocity than the star. This led to magnetic field configurations which varied as indicated very schematically in Figure 4 . Van Ballegooijen started with completely poloidal magnetic field lines (radial in projection on the disk piane), as indicated in the upper panel of Figure 4. As the system wound up, the configuration proceeded to something like the lower panel of Figure 4, where the magnetic field lines got twisted and bulged out over the disk. The energy contained in the magnetic fields increased to an apparent catastrophic point, and Van Ballegooijen assumed that reconnection must take place to reduce the system energy - and start the cycle over again.

The interesting feature of this calculation is that the initial magnetic configuration would be qualitatively favorable to accretion, in that disk material could potentially move along field lines always decreasing its distance from the central star, and thus could be falling "downhill". However, after winding up, the field lines bow outward just above the disk, producing a 

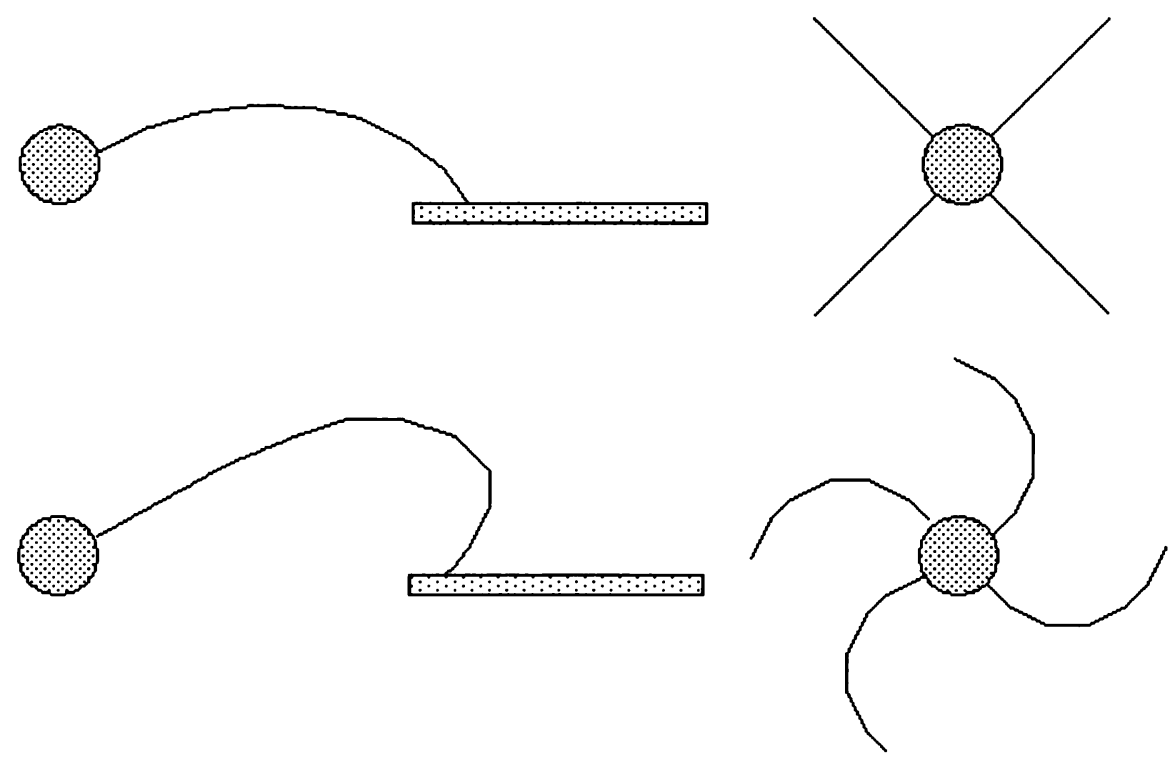

Figure 4. Schematic picture of magnetospheric structure in the situation where the region in the disk in which the stellar magnetic field is rooted has a different angular velocity than the star. The left hand figures show side views, while the right hand figures correspond to top views. Initially poloidal magnetic field lines (top figures) eventually wind up because of the differing angular velocities of the star and the disk region where the magnetic field is fixed (bottom figures). As the field line winds up, the magnetic field strength increases with the increased winding, and the field line bulges out over the disk (lower left). This figure is a schematic representation of the detailed force-free calculations presented by Van Ballegooijen (1994).

potential barrier to accretion (see, e.g., Shu et al. 1994). Thus one might assume that initially untwisted magnetic field lines might allow accretion, but after some twisting accretion would no longer be possible. Moreover, in the configuration shown in the bottom panel of Figure 4, there is an outward force of the magnetic field lines near the disk which would tend to push the disk outward. Thus, one might speculate that a time-dependent model of magnetospheric accretion might show complex behavior, with accretion at times along field lines, which then get twisted up, shut off accretion, and even move outward in the disk to couple to regions outside of corotation. In other words, the same magnetic field line might permit accretion and spindown, as in the Shu et al. (1994) model; however, unlike the Shu et al. model, simultaneous accretion and spindown does not occur, 
but rather alternate. The entire magnetosphere might be a complicated, time-dependent structure, with different field lines originating at differing stellar longitudes and latitudes sometimes allowing accretion, sometimes halting accretion and applying a spindown torque, all in a quasi-random pattern. Reconnection should lead to substantial heating and flare activity; this magnetic heating might be responsible in part for producing high enough temperatures to explain the observed permitted line emission from the magnetospheric gas (Muzerolle et al. 1997).

The implications of such complicated and time-dependent structure on mass ejection are not clear. We need to understand the time-dependence of accretion, and separate it from the rotational modulation of non-axisymmetric magnetic field structure. This will require expanded photometric monitoring programs such as undertaken by Bouvier et al. (1993) and Eaton \& Herbst (1995) before we are able to determine the true timescales of accretion variability in young stellar objects.

Acknowledgements: This work was supported in part by NASA grant NAGW 2306.

\section{References}

Basri, G., \& Bertout, C. 1989, ApJ, 341, 340

Bell, K.R., Lin, D.N.C., Hartmann, L.W., \& Kenyon S.J. 1995, ApJ, 444, 376

Bertout, C., Basri, G., \& Bouvier, J. 1988, ApJ, 330, 350

Bouvier, J., Cabrit, S., Fernandez, M., Martin, E.L., Matthews, J.M. 1993, A\&A, 272, 176

Camenzind, M. 1990, Reviews in Modern Astronomy, (Springer-Verlag: Berlin), 3, 234

Clarke, C.J., Lin, D.N.C., Papaloizou, J.C.B. 1989, MNRAS, 236, 495

Clarke, C.J., Lin, D.N.C., Pringle, J.E 1990, MNRAS, 242, 439

Edwards, S., Strom, S.E., Hartigan, P., Strom, K.M., Hillenbrand, L.A., Herbst, W., Attridge, J., Merrill, K.M., Probst, R., \& Gatley, I. 1993, AJ, 106, 372

Eaton, N.L., \& Herbst, W. 1995, AJ, 110, 2369

Edwards, S., Hartigan, P., Ghandour, L., \& Andrulis, C. 1994, AJ, 108, 1056

Ghosh, P., \& Lamb, F.K. 1979, ApJ, 234, 296

Gullbring, E., Hartmann, L., Briceño, C., \& Calvet, N. 1997, in preparation

Hartigan, P., Hartmann, L., Kenyon, S.J., Strom, S.E., Skrutskie, M.F. 1990, ApJ, 354, $25 \mathrm{~L}$

Hartigan, P., Strom, S., Edwards, S., Kenyon, S., Hartmann, L., Stauffer, J., \& Welty, A. $1991, \mathrm{ApJ}, 382,617$

Hartigan, P., Edwards, S., \& Ghandour, L. 1995, ApJ, 452, 736

Hartmann, L., \& Kenyon, S.J. 1996, ARAA, 34, 207

Hartmann L. 1995, in Circumstellar Disks, Outflows, and Star Formation, Rev. Mex. Astr. Ap. (Serie de Conferencias), 1, 285.

Herbig, G.H. 1977b, ApJ, 217, 693

Herbig, G.H. 1989, in ESO Workshop on Low-Mass Star Formation and Pre-Main Sequence Objects, ed. B. Reipurth (Garching: ESO), 233

Kenyon, S.J., Hartmann, L., Imhoff, C.L., Cassatella, A. 1989, ApJ, 344, 925

Königl, A. 1989, ApJ, 342, 208

Königl, A. 1991, ApJL, 370, L39

Lynden-Bell, D., \& Pringle, J.E. 1974, MNRAS, 168, 603 
Muzerolle, J., Calvet, N., \& Hartmann, L. 1997, in preparation

Najita, J., \& Shu, F.H. 1994, ApJ, 429, 808

Ostriker, E.C., \& Shu, F.H. 1995, ApJ, 447, 813

Popham, R., Narayan, R., Hartmann, L., \& Kenyon, S. 1993, ApJ, 415, L127

Popham, R., Kenyon, S., Hartmann, L., \& Narayan, R. 1996, ApJ, 473, 422

Reipurth, B. 1989, in ESO Workshop on Low Mass Star Formation and Pre-Main Sequence Objects, ed. B. Reipurth (Garching: ESO Conference and Workshop Proceedings No. 33), 247

Reipurth, B. 1991 in The Physics of Star Formation and Early Stellar Evolution, eds. C.J. Lada \& N.D. Kylafis (Dordrecht:Kluwer), 497

Shu, F., Najita, J., Ostriker, E., Wilkin, F., Ruden, S., \& Lizano, S. 1994, ApJ, 429, 781

Shu, F., Najita, J., Ostriker, E.C., \& Shang, H. 1995, ApJ, 455, L155

Valenti, J.A., Basri, G., \& Johns, C.M. 1993, AJ, 106, 2024

Van Ballegooijen, A.A. 1994, Space Sci. Rev., 68, 299

Wang, Y.-M. 1995, ApJ, 449, L153

Wang, Y.-M. 1996, ApJ, 465, L111 